\title{
Electronic traffic signs: the interplay between hybrid and full matrix e-signs
}

\author{
Antonio Lucas-Alba, Ana Hernando Mazón, Ma Teresa Blanch Micó \\ Facultad de Ciencias Sociales y Humanas, Universidad de Zaragoza, Spain \\ Diego Gutiérrez Pérez, José I. Echevarría Vallespí, Nicolás Landa Tejero-Garcés \\ Grupo de Informática Básica Avanzada, Universidad de Zaragoza, Spain
}

\begin{abstract}
Road signs constitute a complex and growing communication system where different elements (pictograms, shapes, texts, etc.) are combined following different strategies. In this paper we have confronted drivers with a number of messages (congestion or road works, before, between, after location/s) developed as an adaptation of Advance Location Signs (class $\mathrm{G}, 1 \mathrm{c}$ in the 1968 Convention) to electronic displays. We manipulate two main factors a) the reading strategy (top-down vs. bottom-up) and the type of matrix display (hybrid, dissociating pictogram and text, vs. full matrix), in a repeated measures experimental design. The time taken to answer and the response given (correct, incorrect) was measured for each of the 24 message-blocks. Results show that the organization of the elements displayed is a key determinant for driver comprehension. Further thoughts on the need to understand the interplay between the formats adopted by static vs electronic message signs are provided.
\end{abstract}

\section{BACKGROUND}

This paper deals specifically with complex road signs, i.e., road signs made of many elementary parts as pictograms, numbers, abbreviations or words. Compare (elementary) signs A (danger warning) or B (prohibitory) in the 1968 Convention (ECE/TRANS/196, 2007), with (compound) signs G, 1 (advance direction signs) or G, 15 (road open or closed). Modern electronic road signage (e-signs), either in-car or VMS, may display both. But esigns main raison d'être is fulfilling functions no other road signs could, e.g., locating variable events ahead, diversions and detours, or strategic truck parking. We will focus here on complex road signs showing the qualitative location of variable events.

\subsection{The official, available templates and display matrices}

The Consolidated Resolution on Road Signs and Signals (ECE/TRANS/212, 2010) recommends the 1968 Convention G, 1 signs to display direction, position or indication on VMS. Hence, we adopt here the G, 1 class (Advance Direction Signs, ADS) as the main templates to follow when designing qualitative location of variable events (e.g., road works between City A and City B) on VMS. We will call them Advance Location Signs (ALS). Furthermore, the 1968 Convention offers some hints on the way forward (p. 51): 
"Advance direction signs G, 1 may bear the symbols used on other signs informing road users of the characteristics of the route or of traffic conditions (for example: signs A, 2; A, 5; C, 3e; C, 6; E, 5a; F, 2).”

This paper will focus on the official G, 1c template to build up the corresponding ALS (Fig. 1). The main reason is that $\mathrm{G}, 1 \mathrm{c}$ is a stack sign (Lay, 2004) and is read as a verbal text (from left to right, and from top to bottom, see Chan \& Spalek, 2005). Standard (hybrid) VMS are also read as text (Ellenberg \& Fabre, 1995). For a number of technical and economic reasons (Haitz \& Tsao, 2011), hybrid VMS split two zones, graphic (pictogram) and alphanumeric (text), downgrading these signs from international to local. Our experience on VMS harmonization, however, point to certain design strategies that can make some displays on hybrid VMS nearly or fully international (Arbaiza \& Lucas, 2012). On the other hand, the coming generation of full matrix VMS and in-car devices may reduce differences with paint coat by means of a more generous LED surface. We aim to compare the differences in comprehension between hybrid VMS and ALS based on G, 1c.

\subsection{Building ALS based on G, 1c and the corresponding hybrid VMS}

How to locate variable events having $\mathrm{G}, 1 \mathrm{c}$ as a basic model? G, 1c makes the most of standard (western) reading patterns. The location above comes first (Northchurch), the one below comes afterwards (Wiggington). Numbers (miles) reinforce that reading pattern and disambiguate other possibilities. Hence ALS should be obtained by the interpolation of the variable events taking into account these reading patterns. Fig. 1 shows an example taking the upper box of G, 1c as a guide. Provided that our electronic sign is full matrix, we may write messages as shown in the center column. An additional question regarding the use of numbers in todays' VMS is: would that numbers be taken as miles or as minutes? For reasons discussed later, we are comparing messages with the same number of informative elements: arrow, toponyms, and pictogram (i.e., numbers are removed).

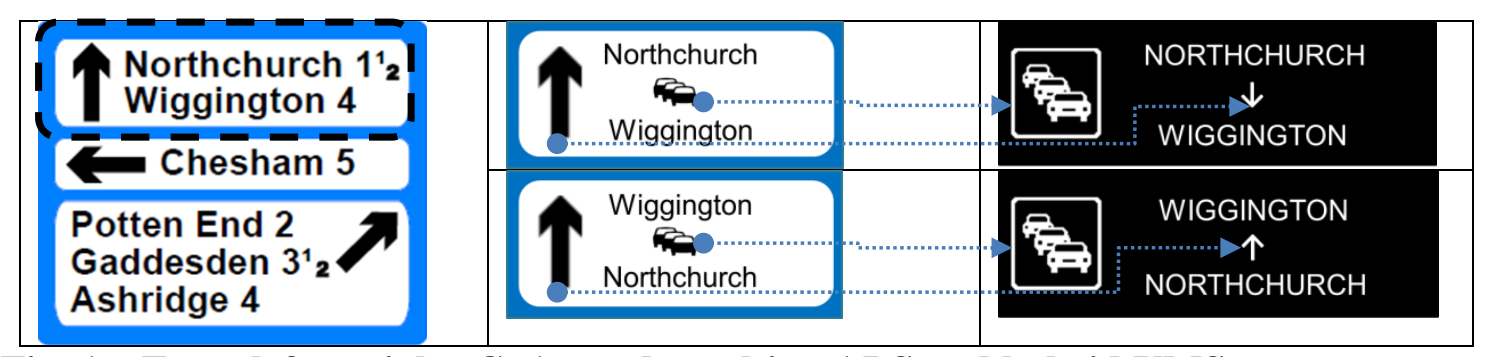

Fig. 1 - From left to right, G, 1c, and resulting ALS and hybrid VMS

Hybrid VMS can only display pictograms on the graphic part. They cannot follow the full logic of road signs design available in the 1968 Convention. So the four basic elements in Fig. 1 (congestion, toponyms and arrow) must be somehow re-arranged. As a result, hybridVMS can only display certain messages adopting an international structure. Fig. 1 shows the basic movements that would bring a) a stack sign model G, 1c to b) a would-be ASL adapted 
for a full-matrix VMS and c) to a hybrid-VMS. This paper will explore empirically the following issues:

- The soundness of the verbal reading pattern: if the left-right/top-down scanning actually leads the reading, comprehension should yield better results when the first location on the way is placed above and the second below, than vice versa.

- The soundness of the full matrix template: placing congestion between, above or below locations following the $\mathrm{G}, 1 \mathrm{c}$ template should outperform hybrid VMS.

- Relative difficulties of specific locations: locating an event that happens between and towards a city will be better understood than locating an event happening after the city, particularly on hybrid VMS (see Fig. 3).

- Anchoring the route beforehand: participants being asked to tell the order of the cities with regards to which events may be located, will have better and swifter comprehension rates.

- Message facilitation: in a manner similar to repetitive or semantic priming (Crundall \& Underwood, 2001) presenting a similar, complementary message before the target message will yield better results than controls.

\section{METHOD}

\subsection{Design}

The design was a mixed model. The anchoring of the route (present, absent) was the between-subjects factor. Twenty four blocks of messages were shown at random to participants. With regards to the blocks, the scanning disposition (top-down vs bottom-up), the type of VMS layout (full matrix vs hybrid), the event location (before, between, after) and the presence of a prior complementary message (present, absent), were within-subject factors. Dependent measures form two sets: response correctness and time to answer. The complementary messages showed a different matrix structure (either hybrid or full matrix) but followed the same reading order (either top-down or bottom-up), and also displayed the same pictogram and city names as targets (Fig. 3).

\subsection{Participants}

The sample was composed of 50 people ( 26 women), whose average age was 28.2 years (SD $=9.55)$. The basic requirement was having passed the theoretical driving test, although $58 \%$ of participants had a driving license. The sample presented a medium-high education level (50\% were university graduates, $20 \%$ had vocational training, $14 \%$ were high school graduates and $16 \%$ had elementary education). Among them, $75.9 \%$ had more than 5 years of driving experience and $62.1 \%$ drive more than $10,000 \mathrm{~km}$ a year. They were asked how often they drove by highway/motorway with a scale from 1 (never) to 3 (often), being the $62 \%$ with driving license who indicated "often".

\subsection{Materials}

The study was carried out in a driving school of a Spanish city. The study cursed on a quiet 
room, in adequate sound, light and temperature conditions for the task execution. The stimulus were presented by means of MediaLab Software (v. 2014) using a laptop computer (HP EliteBook 840, 4 GB of RAM, Intel Core Processor i5-6200U with Intel HD Graphics 520 (2.3GHz), and Windows 7 Professional 64 operating system). The laptop was connected to a 22-inch screen (Philips, screen resolution 1920x1080 and $59 \mathrm{~Hz}$ ) to show the stimuli. A precision Apple USB keyboard (PCB DirectIn V2012) was also used. The participant sat on a comfortable office chair in a desk.

\subsection{Procedure}

Recruitment was arranged through a direct invitation in the driving school. On the test day each participant was provided with an informed consent card, which was read (and signed). The next step was the experimental block itself. The preliminary phase consisted on the answer to demographic questions. Then participants were told to imagine they were driving in a road from Guadalajara to Getafe, passing through Alcalá, Torrejón, and Coslada (Fig. 2). Then, half of the participants were immediately required to indicate the sequence formed by cities (Guadalajara was placed and they chose the order from a list). The same task followed for all participants at the end of the experiment.

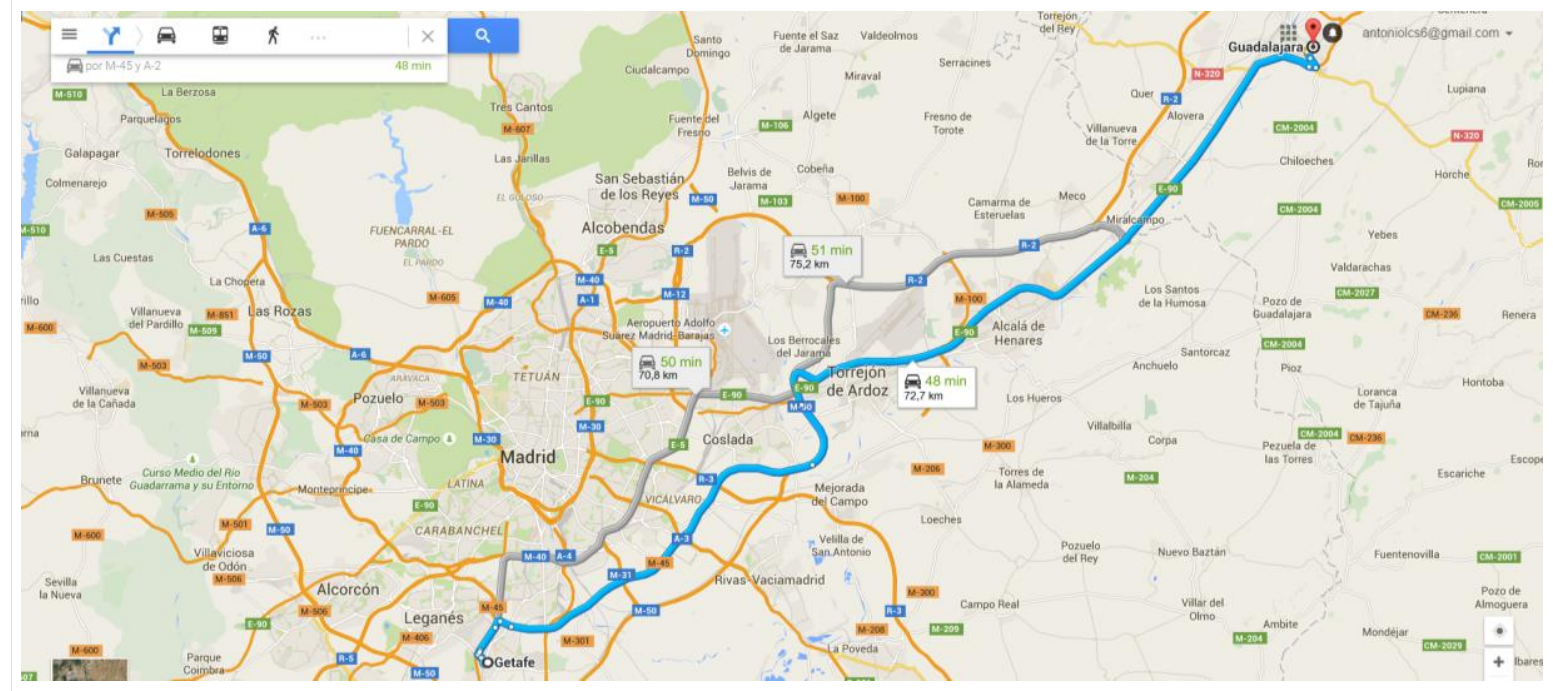

Fig. 2 - The route where the events leading to displaying ALS would happen

The experimental phase proper consisted on 24 blocks of 2 messages presented at random (Fig. 3). All participants answered to three example items first. Participants were told to obviate the first message and answer always with regards to the second one. They were required to indicate where the event was placed with regards to the cities displayed. There were two options to answer, pressing one of two keys on the keyboard. The messages displaying two locations had two options (1 was for "before city A", and 2 was for "before city B"). Messages displaying one location also had two options (1 was for "after city A" and 2 for "before city A" (alternative after/after and before/after options were also available). All the stimuli were located in the center of the screen. The e-signs displayed occupied an area of $1024 \times 290$ pixels. Firstly, a fixation cross was shown for $500 \mathrm{~ms}$. Then the first 
message (complementary message vs control) was shown $2 \mathrm{~s}$. Then another fixation cross was shown for $500 \mathrm{~ms}$. Then the target message was shown for $4 \mathrm{~s}$. SOA was $2500 \mathrm{~ms}$. The screen was shown in black during $3 \mathrm{~s}$ between blocks.

\begin{tabular}{|c|c|c|c|c|}
\hline & \multicolumn{2}{|c|}{ Top-down } & \multicolumn{2}{|c|}{ Bottom-up } \\
\hline Location & Hybrid VMS & Full matrix & Hybrid VMS & Full matrix \\
\hline Toward & TORREJON & TORREJ & $\sum_{\substack{\text { TORRJON } \\
\uparrow}}$ & 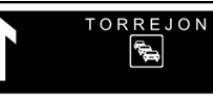 \\
\hline Between & $\notin \begin{array}{c}\text { ALCALA } \\
\text { coslada }\end{array}$ & & $\begin{array}{c}\text { GETAFE } \\
\uparrow \\
\text { TOREJON }\end{array}$ & $\begin{array}{l}\text { GETAFE } \\
\text { TORREJON } \\
\text { TOR }\end{array}$ \\
\hline After & $\underset{\downarrow}{\cos L A D A}$ & SLADA & $\stackrel{\uparrow}{\cos L A D A}$ & $\begin{array}{c}\text { cosLADA } \\
\text { Cos }\end{array}$ \\
\hline
\end{tabular}

Fig. 3 - Basic message set (examples with road works and congestion)

\section{RESULTS}

\subsection{Descriptive}

Table 1 presents the averages and standard deviations of skin conductance for the whole sample separated by driving technique.

\begin{tabular}{|c|c|c|c|c|c|}
\hline & & \multicolumn{2}{|c|}{ Correct } & \multicolumn{2}{c|}{ Time (ms) } \\
\hline Factor & Level & $\mathrm{M}$ & $\mathrm{SD}$ & $\mathrm{M}$ & $\mathrm{SD}$ \\
\hline \multirow{2}{*}{ Route anchorage } & Control & .59 & .018 & 2815 & 231.91 \\
\cline { 2 - 6 } & Present & .64 & .016 & 3500 & 214.04 \\
\hline \multirow{2}{*}{ Reading format } & Top-down & .54 & .019 & 3265 & 182.60 \\
\cline { 2 - 6 } & Bottom-up & .70 & .023 & 3050 & 175.08 \\
\hline \multirow{2}{*}{ Matrix type } & Hybrid & .67 & .020 & 3072 & 166.72 \\
\cline { 2 - 6 } & Full matrix & .56 & .015 & 3242 & 183.29 \\
\hline \multirow{2}{*}{$\begin{array}{c}\text { Complementary } \\
\text { message }\end{array}$} & Present & .63 & .018 & 2923 & 171.93 \\
\cline { 2 - 6 } Event location & Control & .60 & .016 & 3392 & 183.18 \\
\cline { 2 - 6 } & Before & .69 & .027 & 2830 & 177.52 \\
\cline { 2 - 6 } & Between & .69 & .020 & 3824 & 210.27 \\
\cline { 2 - 6 } & After & .46 & .035 & 2819 & 193.04 \\
\hline
\end{tabular}

Table 1 -Measures of correctness and response time

\subsection{Inferential analysis}

\subsubsection{Correct answers}

A mixed ANOVA has been carried on for a 2 (route anchor present /control) x 2 (reading format top-down / bottom-up) x 2 (matrix type hybrid / full) x 2 (complementary message present / control) x 3 (event location before / between / after) design. Only the first factor is 
between-subjects. The analyses show significant differences in the effect of the initial route anchor, $F_{(1,48)}=5.24 ; \mathrm{p}<.05, \eta_{\mathrm{p}}{ }^{2}=0.098$. There is also an effect of the reading format, $F_{(1,48)}=21.23 ; \mathrm{p}<.001, \eta_{\mathrm{p}}{ }^{2}=0.301$, yielding more correct answers when messages are written bottom-up. There is also an effect of the matrix type, $F_{(1,48)}=20.86 ; \mathrm{p}<.001, \eta_{\mathrm{p}}{ }^{2}=$ 0.303 , hybrid VMS yielding more correct answers. Both factors yield an interaction, $F_{(1,48)}$ $=10.69 ; \mathrm{p}<.01, \eta_{\mathrm{p}}{ }^{2}=0.182$ : when reading bottom-up the differences between matrix type are not so acute (hybrid: $M=0.71$; full: $M=0.64$ ) as when reading top-down (hybrid: $M=$ 0.63; full matrix: $M=0.44$ ).

Mauchly's W test indicates that the assumption of sphericity is not reached for the factor grouping event location $\left(X_{(d f=2)}^{2}=26,14, p<0,001\right.$, Chi-square). Therefore the degrees of freedom were corrected using Greenhouse-Geisser estimates of sphericity $(\varepsilon=0.701)$, quite conservative (Pardo \& San Martín, 2010). The analyses show significant differences in event location, $F_{(2,96)}=19.17 ; \mathrm{p}<.001, \eta_{\mathrm{p}}{ }^{2}=0.285$. More mistakes are made when the event is located after a city than before or between two cities, $F_{(1,48)}=22.28$; p .001, quadratic (table 1). Event location yields a (marginally significant) interaction with matrix type, $\mathrm{F}_{(2,96)}=2,85$; $\mathrm{p}=0,066, \eta_{\mathrm{p}}^{2}=0,056$, and this gives way to a second order interaction with reading format (top-down, bottom-up), $F_{(2,96)}=3.34 ; \mathrm{p}<.05, \eta_{\mathrm{p}}^{2}=0.065$ (Fig. 4).
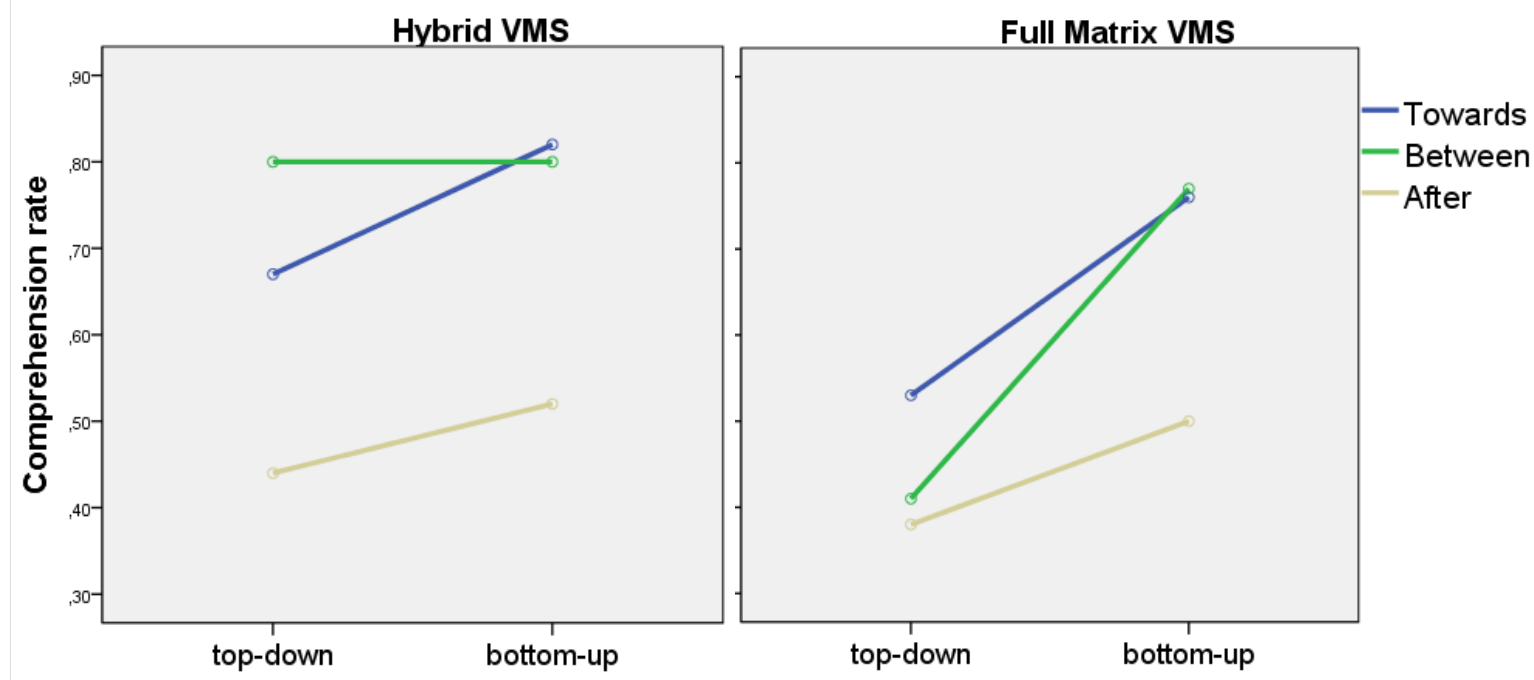

Fig. 4 - Reading format, matrix type and location mean correctness

\subsubsection{Response time}

The analyses show significant differences in the effect of the initial route anchor, $F_{(1,48)}=$ $4.71 ; \mathrm{p}<.05, \eta_{\mathrm{p}}{ }^{2}=0.089$ (table 1 ). There is also an effect of the complementary message, $F_{(1,48)}=8.28 ; \mathrm{p}<.01, \eta_{\mathrm{p}}{ }^{2}=0.147$, the previous display of a complementary message yields lower response times than controls. The absence/presence of a complementary message nuances the effect of the reading format, $F_{(2,96)}=11.47 ; \mathrm{p}<.001, \eta_{\mathrm{p}}{ }^{2}=0,193$ : the presence $(M=3306)$ or absence $(M=3225)$ of a top-down complementary message yields similar response times compared to the presence $(M=2540)$ /absence $(M=3560)$ of the bottom-up one. Finally, the analyses show significant differences in event location, $F_{(2,96)}=17.41 ; \mathrm{p}<$ 
$.001, \eta_{\mathrm{p}}{ }^{2}=0.266$. Locating events between two cities (four elements) takes more time to answer than locating before or after one city, $F_{(1,48)}=29.79 ; \mathrm{p}<.001$, quadratic (table 1 ).

\section{DISCUSSION}

Official road signs (ECE/TRANS/196, 2007) make use of two main design trends, either staking information (top-down) or presenting it as a diagram (Lay, 2004). Compared to hybrid VMS, full matrix e-signs allow for certain possibilities (e.g., inserting a pictogram between locations). We have followed the G, 1c template to make a full matrix e-sign (ALS), although not completely: we have not placed numbers (referring to miles or kilometers). We made it this way for two reasons, a) to keep the very same number of informative elements on each message (parsimony) and b) because our experience in previous comprehension test tells us that isolated numbers on VMS displays are not self-evident as in posted ADS. Drivers may think that numbers are travel times, exits or speeds.

Results indicate that the information displayed top-down obtains poor comprehension, particularly with full matrix displays. Stacked information may work if, following the immediacy principle (cf. Carroll, 2008), each element on the stack is understood and inserted in a phrase or sentence structure. Hybrid VMS seem to follow this mechanism. For example, location between cities is similarly understood either when the arrow points up or down, and this is more or less the case for location towards city (Fig. 3). The problem is locating the event after city and the meaning assigned to the arrow (Kurata \& Egenhoffer, 2005). For example, considering the bottom-up display, the element "arrow" on the stack may have two meanings: after/from and in the direction of (quite common for any arrow). The first translation would yield a good top-down answer, but the second translation of the arrow equates after and towards (in fact, this is a common result in standard comprehension tests; EIP, 2015). Here ambiguity plays against coherence. The arrow pointing up (or down) to toponyms may also be translated as towards or as in the direction of, i.e., both agree on message meaning. But full matrixes made after the $G$, 1c template behave more ambiguously. Drivers understand them but only bottom-up. The big arrow on the left means "in the direction of" or "towards there" but it also seems to adopt a quasi-diagrammatic stance -i.e., the arrow represents the road. Locations are so read in parallel to that road: up is far, down is near, and events are located in correspondence. The size of the arrow prevents the verbal reading scheme, although not completely. Adding numbers representing miles or kilometers is more an orthopedic measure than required information. This context may explain why complementary information between hybrid and full matrix displays, previous to the target message, had no effect on comprehension.

However, being exposed $2 \mathrm{~s}(\mathrm{SOA}=2.5 \mathrm{~s})$ to a bottom-up message improves response time of the corresponding target (nearly one second compared to the control) with hybrid VMS and full matrix designs the same. The human mind may translate into a mental model both written and visual information (Johnson-Laird, 2006). What type of mental representation 
enhanced the complementary message? Complex representations as such are not stored in Long Term Memory (perhaps this is one reason for not finding a facilitator effect on comprehension). This issue, as well as the overall effects observed by anchoring of the routes (enhancing comprehension, but slowing response times) open new ways for our enquiry, concerning the operative modes followed to understand signs, and the role of both short and long term memory on the mental manipulation and response to road information.

\section{AKNOWLEDGEMENTS}

This paper has been done with the support of the Dirección General de Tráfico, Spain, under grant SPIP2014-01345: Análisis de las claves de desarrollo de los criterios de diseño de la señalización variable teniendo en cuenta el factor de su internacionalización, presente y futura.

\section{REFERENCES}

ARBAIZA, A., LUCAS, A. (Coords.) (2012). VMS-DG01. Principles of VMS Message Design. Version 2.0, December 2012. http://www.easyway-its.eu/

CARROLL, D.W. (2008). Psychology of Language. Belmont: Thomson.

CHAN, T.T., BERGEN, B. (2005). Writing direction influences spatial cognition. In B. Bara, L. Barsalou and M. Bucciarelli (Eds.). Proceedings of the $27^{\text {th }}$ Annual Conference of the Cognitive Science Society Mahwah, NJ: Erlbaum, 412-417.

CRUNDALL, D., UNDERWOOD, G. (2001). The priming function of road signs. Transportation Research Part F, 4, 187-200.

ECE/TRANS/196 (2007). Convention on Road Signs and Signals of 1968. United Nations: New York and Geneva.

ECE/TRANS/212 (2010). Consolidated Resolution on Road Signs and Signals (R.E.2). http://www.unece.org/trans/roadsafe/rsrec.html

EIP (2015). Evaluation of the use of the procedures and knowledge developed for VMS harmonization in concrete applications. Deliverable 1. SA4.2 VMS Harmonization. European ITS Platform. https://www.easyway-its.eu/

ELlENBERG, M., FABRE, P. (1995). Melyssa Corridor. Mediterranean Lyon-Sttutgart Site for ATT. Transport Telematics Project V2040. D10. Commission of the European Communities - R\&D Programme Telematics Systems in the Area of Transport (DRIVE II). HAITZ, R., TSAO, J.Y. (2011). Solid-state lighting: 'The case' 10 years after and future prospects. Physica Status Solidi A 208, No. 1, 17-29.

JOHNSON-LAIRD, P. N. (2006). How we reason. New York: Oxford University Press.

KRAMPEN, M. (1983). Icons of the road. Semiotica, 43 (1/2), 1-203.

KURATA, Y., EGENHOFER, M. (2005). Structure and Semantics of Arrow Diagrams. COSIT '05, September 2005, Ellicottville, NY.

LAY, M.G. (2004). Design of traffic signs. In C. Castro and T. Horberry (Eds.): The Human Factors of Transport Signs, CRC Press, Boca Raton, 25-48. 